\title{
THE EXTERNAL AXISYMMETRIC INTERFACE CRACK WITH HEAT FLOW
}

\author{
By J. R. BARBER and MARIA COMNINOU
}

(Department of Mechanical Engineering and Applied Mechanics and the Department of Civil Engineering, University of Michigan, Ann Arbor, MI 48109, U.S.A.)

[Received 8 May 1981. Revise 17 November 1981]

\section{SUMMARY}

Two half-spaces of dissimilar material properties are brought together and bonded over a circular region of radius $r=a$ to form an exterior axisymmetric interface crack. Loads are applied at infinity such that the common boundary (i.e. the bond and any contact region that develops) transmits tractions whose resultant is an axial tension $P$. Interpenetration is predicted if the crack is assumed to be completely open; in fact an annular contact region $(a<r \leqslant b)$ is developed around the bond, while separation occurs in $r>b$. If the temperatures of the bodies are now changed, the extent of this contact region changes.

A solution to this problem is obtained by representing the displacement in the bodies in terms of harmonic potential functions and reducing the resulting mixed boundary-value problem to an integral equation which is solved numerically. Detailed results are given for the particular case in which both bodies are raised to the same temperature. If the load $P$ is zero, the crack either closes completely or opens almost completely, depending on the sign of certain combinations of physical constants.

Results are also given for the case where one body is a rigid perfect conductor and the other is maintained at zero temperature.

\section{Introduction}

IF two half-spaces of dissimilar materials are bonded together along part of their common interface and loads are applied tending to separate the bond, the assumption that the unbonded region opens everywhere leads to a solution involving oscillatory singularities and interpenetration near the crack tips $(1,2)$. However, a physically correct solution can be obtained by admitting the existence of a contact region between the separation and bonded regions.

In this paper, we shall consider the problem of an axisymmetric external interface crack in which stresses are developed due to variations in temperature as well as external loading. We restrict attention to the case in which heat flows into the more distortive material to avoid the difficulties associated with the concept of imperfect contact (3). Detailed results are given for the limiting case where both bodies are raised to the same temperature. 


\section{Statement of the problem}

Two isotropic elastic half-spaces are bonded over the circular region $0 \leqslant r \leqslant a$ at their common plane $z=0$ and are free of stress when the temperature is everywhere zero. The suffixes 1,2 will be used to refer to the bodies $z>0, z<0$ respectively.

Those regions of the two bodies distant from the bond are now raised to different temperatures $T_{1}^{\infty}, T_{2}^{\infty}$, causing heat to flow through the bond and through any other region of the interface at which mechanical contact occurs. We assume that no heat flow occurs in regions of separation and that there is no interfacial thermal resistance in the contact region. The bodies are simultaneously pulled apart by a force $P$.

Experience with 'athermal' interface crack problems (2) suggests that the force $P$ will separate the bodies in most of the unbounded region of the interface, but cause a small annulus of contact surrounding the bond. This type of behaviour extends to the thermoelastic case. We denote the outer radius of the contact annulus by $b$ and assume the contact to be frictionless.

\section{Mathematical formulation}

It is mathematically convenient to regard the solution for each half-space as the sum of a uniform temperature rise $T_{1}^{\infty}, T_{2}^{\infty}$, and a perturbation with temperature tending to zero at infinity. The uniform temperature component will produce a uniform dilatation but no thermal stress or heat flux.

For the perturbation in each half-space, we use a solution of the equations of thermoelastic equilibrium in terms of three harmonic functions derived from solutions $A, B$ of Green and Zerna (4) and solution $B$ of (5). The displacement and temperature are

$$
\begin{gathered}
\mathbf{a}=\frac{(1-\nu)}{\mu} \nabla \phi+\frac{z}{2 \mu} \nabla \frac{\partial \phi}{\partial z}-\frac{(3-4 \nu)}{2 \mu} \mathbf{k} \frac{\partial \phi}{\partial z}+2 \delta \mathbf{k} \frac{\partial \psi}{\partial z}-\delta \nabla \psi+ \\
+\frac{(1-2 \nu)}{2 \mu} \nabla \omega+\frac{z}{2 \mu} \nabla \frac{\partial \omega}{\partial z}-\frac{(3-4 \nu)}{2 \mu} \mathbf{k} \frac{\partial \omega}{\partial z} \\
T=\frac{1}{K} \frac{\partial^{2} \psi}{\partial z^{2}},
\end{gathered}
$$

where

$$
\begin{gathered}
\nabla^{2}(\phi, \omega, \psi)=\mathbf{0}, \\
\delta=\frac{\alpha(1+\nu)}{K}
\end{gathered}
$$

is the thermal distortivity and $\alpha, K$ are respectively the coefficients of thermal expansion and thermal conductivity. 
The stresses at the plane $z=0$ take the form

$$
\begin{gathered}
\sigma_{z z}=-\frac{\partial^{2} \omega}{\partial z^{2}}, \quad \text { on } \quad z=0 \\
\sigma_{r z}=\frac{\partial^{2} \phi}{\partial r \partial z}, \quad \text { on } \quad z=0
\end{gathered}
$$

and hence we can satisfy the continuity conditions

$$
\begin{aligned}
& \sigma_{z z 1}=\sigma_{z z 2}, \text { on } z=0, \\
& \sigma_{r z 1}=\sigma_{r z 2},
\end{aligned}
$$

by defining

$$
\begin{gathered}
\omega(r, z) \equiv \omega_{1}(r, z)=\omega_{2}(r,-z) ; \\
\phi(r, z) \equiv \phi_{1}(r, z)=-\phi_{2}(r,-z) .
\end{gathered}
$$

In other words, the functions $\phi_{2}, \omega_{2}$ in body 2 are respectively odd and even continuations into $z<0$ of $\phi_{1}, \omega_{1}$. Note, however, that no conditions of continuity are imposed at $z=0$ on the functions $\phi$ and $\omega$.

The continuity condition on heat flux

$$
q_{z 1}=q_{z 2} \text { on } z=0,
$$

is satisfied in the same way by defining

$$
\psi(r, z) \equiv \psi_{1}(r, z)=-\psi_{2}(r,-z),
$$

since

$$
q_{z}=-K \frac{\partial T}{\partial z}=-\frac{\partial^{3} \psi}{\partial z^{3}}
$$

from equation (2).

In each region of the interface there are three further boundary conditions which define a boundary-value problem for the functions $\omega, \phi, \psi$ in $z>0$.

In the bonded region, we have continuity of temperature and of both normal and radial displacement, so that

$$
\left.\begin{array}{l}
T_{1}=T_{2}, \\
u_{z 1}=u_{z 2}, \\
u_{r 1}=u_{r 2},
\end{array}\right\}, 0 \leqslant r \leqslant a .
$$

In the contact region, we have continuity of temperature and normal displacements, and the shear stresses vanish for frictionless contact, so that

$$
\left.\begin{array}{rl}
T_{1} & =T_{2}, \\
u_{z 1} & =u_{z 2}, \\
\sigma_{r z} & =0,
\end{array}\right\}, a<r \leqslant b .
$$


Finally, in the separation region, both normal and shear tractions are zero and there is no heat flux, i.e.

$$
\left.\begin{array}{rl}
q_{z} & =0, \\
\sigma_{r z} & =0, \\
\sigma_{z z} & =0,
\end{array}\right\}, r>b .
$$

\section{Reduction to a problem for the half space}

The condition of temperature continuity (equations $(14,17)$ ) can be written

$$
T_{1}^{\infty}+\frac{1}{K_{1}} \frac{\partial^{2} \psi_{1}}{\partial z^{2}}=T_{2}^{\infty}+\frac{1}{K_{2}} \frac{\partial^{2} \psi_{2}}{\partial z^{2}}, \quad 0 \leqslant r \leqslant b,
$$

since the temperature perturbations (equation (2)) are defined relative to the temperatures at infinity $\left(T_{1}^{\infty}, T_{2}^{\infty}\right)$ in the two bodies.

If we now use (12) and rearrange, we have (on $z=0$ )

$$
\frac{\partial^{2} \psi}{\partial z^{2}}=K\left(T_{2}^{\infty}-T_{1}^{\infty}\right), \quad 0 \leqslant r \leqslant b,
$$

where

$$
K=\frac{K_{1} K_{2}}{\left(K_{1}+K_{2}\right)} .
$$

At the surface $z=0$, the displacement has the components

$$
\begin{aligned}
& u_{z}=-\frac{(1-2 \nu)}{2 \mu} \frac{\partial \phi}{\partial z}-\frac{(1-\nu)}{\mu} \frac{\partial \omega}{\partial z}+\delta \frac{\partial \psi}{\partial z}, \\
& u_{r}=\frac{(1-\nu)}{\mu} \frac{\partial \phi}{\partial r}+\frac{(1-2 \nu)}{2 \mu} \frac{\partial \omega}{\partial r}-\delta \frac{\partial \psi}{\partial r},
\end{aligned}
$$

from (1). Hence, for continuity of normal displacement (equations $(15,18)$ ) we have

$$
\begin{aligned}
-\frac{\left(1-2 \nu_{1}\right)}{2 \mu_{1}} \frac{\partial \phi_{1}}{\partial z}-\frac{\left(1-\nu_{1}\right)}{\mu_{1}} \frac{\partial \omega_{1}}{\partial z} & +\delta_{1} \frac{\partial \psi_{1}}{\partial z} \\
& =-\frac{\left(1-2 \nu_{2}\right)}{2 \mu_{2}} \frac{\partial \phi_{2}}{\partial z}-\frac{\left(1-\nu_{2}\right)}{\mu_{2}} \frac{\partial \omega_{2}}{\partial z}+\delta_{2} \frac{\partial \psi_{2}}{\partial z}+C_{1},
\end{aligned}
$$

where $C_{1}$ is an arbitrary constant permitting a relative rigid-body displacement along the axis. Using equations $(9,10,12),(28)$ can be written in the form

$$
-B \frac{\partial \phi}{\partial z}-A \frac{\partial \omega}{\partial z}+\left(\delta_{1}-\delta_{2}\right) \frac{\partial \psi}{\partial z}=C_{1},
$$


where

$$
\begin{aligned}
& A=\frac{\left(1-\nu_{1}\right)}{\mu_{1}}+\frac{\left(1-\nu_{2}\right)}{\mu_{2}}, \\
& B=\frac{\left(1-2 \nu_{1}\right)}{2 \mu_{1}}-\frac{\left(1-2 \nu_{2}\right)}{2 \mu_{2}} .
\end{aligned}
$$

In developing a similar result for continuity of radial displacement (equation (16)) we note that the uniform temperature rises $T_{1}^{\infty}, T_{2}^{\infty}$ in the two bodies will produce radial displacements $\alpha_{1} T_{1}^{\infty} r, \alpha_{2} T_{2}^{\infty} r$ which must be added to the perturbation solution (27). Hence we have

$$
\begin{aligned}
\frac{\left(1-\nu_{1}\right)}{\mu_{1}} \frac{\partial \phi_{1}}{\partial r}+\frac{\left(1-2 \nu_{1}\right)}{2 \mu_{1}} \frac{\partial \omega_{1}}{\partial r} & -\delta_{1} \frac{\partial \psi_{1}}{\partial r}+\alpha_{1} T_{1}^{\infty} r \\
= & \frac{\left(1-\nu_{2}\right)}{\mu_{2}} \frac{\partial \phi_{2}}{\partial r}+\frac{\left(1-2 \nu_{2}\right)}{2 \mu_{2}} \frac{\partial \omega_{2}}{2 \mu_{2}}-\delta_{2} \frac{\partial \psi_{2}}{\partial r}+\alpha_{2} T_{2}^{\infty} r,
\end{aligned}
$$

which reduces to

$$
A \frac{\partial \phi}{\partial r}+B \frac{\partial \omega}{\partial r}-\left(\delta_{1}+\delta_{2}\right) \frac{\partial \psi}{\partial r}=\left(\alpha_{2} T_{2}^{\infty}-\alpha_{1} T_{1}^{\infty}\right) r
$$

using equations $(9,10,12)$.

We use these results to formulate a mixed boundary-value problem for $\phi$, $\omega, \psi$ in the half-space $z>0$ through the following boundary conditions on $z=0$ :

$$
\frac{\partial^{2} \psi}{\partial z^{2}}=K\left(T_{2}^{\infty}-T_{1}^{\infty}\right), \quad 0 \leqslant r \leqslant b,
$$

from $(14,17,24)$,

$$
\frac{\partial^{3} \psi}{\partial z^{3}}=0, \quad r>b
$$

from $(13,20)$,

$$
A \frac{\partial \phi}{\partial r}+B \frac{\partial \omega}{\partial r}-\left(\delta_{1}+\delta_{2}\right) \frac{\partial \psi}{\partial r}=\left(\alpha_{2} T_{2}^{\infty}-\alpha_{1} T_{1}^{\infty}\right) r, \quad 0 \leqslant r \leqslant a
$$

from $(16,33)$,

$$
\frac{\partial \phi}{\partial z}=0, \quad r>a,
$$

from $(6,19,22)$,

$$
-B \frac{\partial \phi}{\partial z}-A \frac{\partial \omega}{\partial z}+\left(\delta_{1}-\delta_{2}\right) \frac{\partial \psi}{\partial z}=C_{1}, \quad 0 \leqslant r \leqslant b,
$$


from $(15,18,29)$, and

$$
\frac{\partial^{2} \omega}{\partial z^{2}}=0, \quad r>b,
$$

from $(5,22)$.

With reference to equation (37), we note that the condition $\sigma_{r z}=0$ in $r>a, z=0$, strictly only demands $\partial^{2} \phi / \partial r \partial z=0$ and hence permits the solution $\partial \phi / \partial z=$ constant. However, the introduction of such a constant proves to be equivalent to the addition of a further rigid-body displacement $u_{z}$, which can be subsumed under $C_{1}$ in equation (38), thereby preserving the local nature of the solution for $\phi$.

\section{Solution strategy}

The conditions $(34,35)$ involve $\psi$ only, permitting this function and hence the temperature field to be fully determined. We then use the method of Green (4) and Collins (6) to choose representations for $\phi, \omega$ which identically satisfy equations $(37,39)$. The remaining equations $(36,38)$ then furnish two simultaneous integral equations for these functions which are reduced to a single Fredholm equation.

(i) Solution for $\psi$

The conditions $(34,35)$ define a classical mixed boundary-value problem for the function $\partial^{2} \psi / \partial z^{2}$ whose solution is easily shown to be

$$
\frac{\partial^{2} \psi}{\partial z^{2}}=\frac{2 K\left(T_{2}^{\infty}-T_{1}^{\infty}\right)}{\pi} \operatorname{Im}\left[\log \left\{\left(r^{2}+(z+i b)^{2}\right)^{\frac{1}{1}}+z+i b\right\}\right],
$$

where the square root is interpreted in accordance with the conventions of reference (4).

We also require values of $\partial \psi / \partial r$ and $\partial \psi / \partial z$ on $z=0$ for substitution into equations $(36,38)$ and these are conveniently obtained by integration within the plane. Thus, because $\psi$ is harmonic we have

$$
\frac{\partial^{2} \psi}{\partial r^{2}}+\frac{1}{r} \frac{\partial \psi}{\partial r}=-\frac{\partial^{2} \psi}{\partial z^{2}}=-K\left(T_{2}^{\infty}-T_{1}^{\infty}\right), \quad 0 \leqslant r \leqslant b, \quad z=0,
$$

from which, by integration,

$$
\frac{\partial \psi}{\partial r}=-\frac{K\left(T_{2}^{\infty}-T_{1}^{\infty}\right) r}{2}, \quad 0 \leqslant r \leqslant b, \quad z=0,
$$

where an arbitrary constant has been set to zero to preserve continuity at $r=0$.

If we first differentiate (40) with respect to $z$, we can find $\partial \psi / \partial z$ by a 
similar process, obtaining

$$
\begin{gathered}
\frac{\partial \psi}{\partial z}=-\frac{2 K\left(T_{2}^{\infty}-T_{1}^{\infty}\right)}{\pi}\left[\left(b^{2}-r^{2}\right)^{\frac{1}{2}}-b \log \left\{\left(1-r^{2} / b^{2}\right)^{\frac{1}{2}}+1\right\}\right]+C_{2}, \\
0 \leqslant r \leqslant b, \quad z=0,
\end{gathered}
$$

where $C_{2}$ is an integration constant.

(ii) Representation of $\phi, \omega$

Following Green and Zerna (4) and Collins (6) we represent $\partial \phi / \partial z, \partial \omega / \partial z$ in the forms

$$
\begin{aligned}
& \frac{\partial \omega}{\partial z}=\operatorname{Re} \int_{0}^{b} \frac{g_{1}(t) d t}{\left\{r^{2}+(z+i t)^{2}\right\}^{\frac{1}{2}}}, \\
& \frac{\partial \phi}{\partial z}=\operatorname{Im} \int_{0}^{a} \frac{g_{2}(t) d t}{\left\{r^{2}+(z+i t)^{2}\right\}^{\frac{1}{2}}},
\end{aligned}
$$

where $g_{1}, g_{2}$ are real functions of $t$ to be determined.

On $z=0,(45)$ reduces to

$$
\begin{aligned}
\frac{\partial \phi}{\partial z} & =\operatorname{Im} \int_{0}^{a} \frac{g_{2}(t) d t}{\left(r^{2}-t^{2}\right)^{\frac{1}{2}}} \\
& = \begin{cases}0, & r>a, \\
-\int_{r}^{a} \frac{g_{2}(t) d t}{\left(t^{2}-r^{2}\right)^{\frac{1}{2}}}, & 0 \leqslant r \leqslant a,\end{cases}
\end{aligned}
$$

and hence (37) is satisfied identically. We also require the value of $\partial \phi / \partial r$ which is obtained as in section (i) above as

$$
\frac{\partial \phi}{\partial r}=-\frac{1}{r} \int_{0}^{r} \frac{t g_{2}(t) d t}{\left(r^{2}-t^{2}\right)^{\frac{1}{2}}}, \quad 0 \leqslant r \leqslant a, \quad z=0 .
$$

In the same way, it can be shown that (44) satisfies (39) identically and that the other surface values are

$$
\begin{array}{ll}
\frac{\partial \omega}{\partial z}=\int_{0}^{r} \frac{g_{1}(t) d t}{\left(r^{2}-t^{2}\right)^{\frac{1}{2}}}, & 0 \leqslant r \leqslant b, \\
\frac{\partial^{2} \omega}{\partial z^{2}}=\frac{1}{r} \frac{d}{d r} \int_{r}^{b} \frac{t_{1}(t) d t}{\left(t^{2}-r^{2}\right)^{\frac{1}{2}}}, & 0 \leqslant r \leqslant b, \\
\frac{\partial \omega}{\partial r}=\frac{1}{r} \int_{0}^{b} g_{1}(t) d t-\frac{1}{r} \int_{r}^{b} \frac{t g_{1}(t) d t}{\left(t^{2}-r^{2}\right)^{!}}, & 0 \leqslant r \leqslant b .
\end{array}
$$

We note that there are other forms related to $(44,45)$ which satisfy $(37,39)$. The forms chosen are the least restrictive since they permit square-root singularities in the stresses at $r=a, b$, while preserving continuity of displacement for bounded functions $g_{1}(t), g_{2}(t)$. 
(iii) Integral equations for $g_{1}$ and $g_{2}$

We now substitute these expressions into the two remaining boundary conditions $(36,38)$ to obtain integral equations for $g_{1}$ and $g_{2}$.

From (36) we have

$$
\begin{array}{r}
-\frac{A}{r} \int_{0}^{r} \frac{t g_{2}(t) d t}{\left(r^{2}-t^{2}\right)^{\frac{1}{2}}}+\frac{B}{r} \int_{0}^{b} g_{1}(t) d t-\frac{B}{r} \int_{r}^{b} \frac{t g_{1}(t) d t}{\left(t^{2}-r^{2}\right)^{\frac{1}{2}}}+\frac{1}{2}\left(\delta_{1}+\delta_{2}\right) K\left(T_{2}^{\infty}-T_{1}^{\infty}\right) r \\
=\left(\alpha_{2} T_{2}^{\infty}-\alpha_{1} T_{1}^{\infty}\right) r, \quad 0 \leqslant r \leqslant b,
\end{array}
$$

using $(42,49,52)$, which we rearrange as an equation for $g_{2}$ as

$$
\int_{0}^{r} \frac{t g_{2}(t) d t}{\left(r^{2}-t^{2}\right)^{\frac{1}{2}}}=\beta\left[\int_{0}^{b} g_{1}(t) d t-\int_{t}^{b} \frac{t g_{1}(t) d t}{\left(t^{2}-r^{2}\right)^{\frac{1}{2}}}\right]+C_{3} r^{2}, \quad 0 \leqslant r \leqslant b
$$

where

$$
C_{3}=\left\{\frac{1}{2}\left(\delta_{1}+\delta_{2}\right) K\left(T_{2}^{\infty}-T_{1}^{\infty}\right)-\left(\alpha_{2} T_{2}^{\infty}-\alpha_{1} T_{1}^{\infty}\right)\right\} / A,
$$

and

$$
\beta=B / A
$$

is Dundurs' constant (7).

The second equation follows from (38) using $(43,47,48,50)$ and is

$$
\begin{aligned}
& \int_{0}^{r} \frac{g_{1}(t) d t}{\left(r^{2}-t^{2}\right)^{\frac{1}{2}}}= \beta \int_{r}^{a} \frac{g_{2}(t) d t}{\left(t^{2}-r^{2}\right)^{\frac{1}{2}}}+ \\
&+C_{4}-\frac{2 K}{\pi A}\left(T_{2}^{\infty}-T_{1}^{\infty}\right)\left(\delta_{1}-\delta_{2}\right)\left[\left(b^{2}-r^{2}\right)^{\frac{1}{2}}-b \log \left\{\left(1-r^{2} / b^{2}\right)^{\frac{1}{2}}+1\right\}\right], \\
& 0 \leqslant r \leqslant b, \quad(57)
\end{aligned}
$$

where the integral on the right-hand side is to be interpreted as zero in $a \leqslant r \leqslant b$, and $C_{4}$ is a new arbitrary constant replacing $C_{1}$ and $C_{2}$.

(iv) Reduction to a single Fredholm equation

Equations $(54,57)$ can be solved as Abel integral equations for the unknown functions $g_{2}, g_{1}$, respectively, regarding the right-hand sides as known. From (54) we find

$$
\begin{aligned}
g_{2}(x)=\frac{2}{\pi x} \frac{d}{d x} \int_{0}^{x}\left[\beta\left\{\int_{0}^{b} g_{1}(t) d t-\int_{1}^{b} \frac{s g_{1}(s) d s}{\left(s^{2}-t^{2}\right)^{\frac{1}{2}}}\right\}+C_{3} t^{2}\right] \frac{t d t}{\left(x^{2}-t^{2}\right)^{\frac{1}{2}}}, \\
0 \leqslant x \leqslant a,
\end{aligned}
$$

which can be reduced to

$$
g_{2}(x)=\frac{2 \beta x}{\pi} \int_{0}^{b} \frac{g_{1}(s) d s}{\left(x^{2}-s^{2}\right)}+\frac{4 C_{3} x}{\pi}, \quad 0 \leqslant x \leqslant a,
$$

by reversing the order of integration and performing certain integrals. 
A similar sequence of operations on (57) yields the result

$$
\begin{aligned}
g_{1}(x)= & \frac{2 \beta}{\pi} \int_{0}^{a} \frac{\operatorname{tg}_{2}(t) d t}{\left(t^{2}-x^{2}\right)}+\frac{2 C_{4}}{\pi} \\
& -\frac{4 K}{\pi^{2} A}\left(T_{2}^{\infty}-T_{1}^{\infty}\right)\left(\delta_{1}-\delta_{2}\right) b\left\{1-\log 2-\frac{1}{2}(1-x / b) \log (1-x / b)\right. \\
& \left.-\frac{1}{2}(1+x / b) \log (1+x / b)\right\}, \quad 0 \leqslant x \leqslant b .
\end{aligned}
$$

The constant $C_{4}$ can now be determined by imposing the requirement that the contact traction is bounded at the smooth transition from contact to separation at $r=b$. From equations $(5,50)$ we have

$$
\sigma_{z x}=-\frac{1}{r} \frac{d}{d r} \int_{r}^{b} \frac{\operatorname{tg}_{1}(t) d t}{\left(t^{2}-r^{2}\right)^{\frac{1}{2}}}, \quad 0 \leqslant r \leqslant b, \quad z=0,
$$

and hence for $\sigma_{z x}$ to be bounded at $r=b$ we require

from which

$$
g_{1}(b)=0 \text {, }
$$

$$
\frac{2 C_{4}}{\pi}=\frac{2 \beta}{\pi} \int_{0}^{a} \frac{\operatorname{tg}_{2}(t) d t}{\left(b^{2}-t^{2}\right)}+\frac{4 K}{\pi^{2} A}\left(T_{2}^{\infty}-T_{1}^{\infty}\right)\left(\delta_{1}-\delta_{2}\right) b(1-2 \log 2) .
$$

Finally we substitute for $g_{1}, C_{4}$ from equations $(59,62)$ into $(59)$ and use the Poincaré-Bertrand result to invert the order of integration, obtaining

$$
\begin{aligned}
g_{2}(x)\left(1-\beta^{2}\right)= & \frac{4 \beta^{2}}{\pi^{2}}\left[\int_{0}^{a}\left\{\frac{1}{2} x \log \left(\frac{b+t}{b-t}\right)-\frac{1}{2} t \log \left(\frac{b+x}{b-x}\right)\right\} \frac{g_{2}(t) d t}{\left(x^{2}-t^{2}\right)}+\right. \\
& \left.+\int_{0}^{a}\left\{\frac{1}{2} t \log \left(\frac{b+t}{b-t}\right)\right\} \frac{g_{2}(t) d t}{\left(b^{2}-t^{2}\right)}\right]+ \\
& +\frac{4 \beta K}{\pi^{3} A}\left(T_{2}^{\infty}-T_{1}^{\infty}\right)\left(\delta_{1}-\delta_{2}\right) b x \times \\
& \times \int_{0}^{b}\left\{\left(1-\frac{s}{b}\right) \log \left(1-\frac{s}{b}\right)+\left(1+\frac{s}{b}\right) \log \left(1+\frac{s}{b}\right)-2 \log 2\right\} \times \\
& \times \frac{d s}{\left(x^{2}-s^{2}\right)}+\frac{4 C_{3} x}{\pi}, \quad 0 \leqslant x \leqslant a .
\end{aligned}
$$

\section{Interface tractions}

Equation (64) can be cast in a dimensionless form by defining

$$
\begin{aligned}
g(x) & =\frac{A_{g_{2}}(x)}{a}, \\
c & =b / a \quad(>1), \\
y & =x / a,
\end{aligned}
$$


412

J. R. BARBER AND MARIA COMNINOU

in terms of which

$$
\begin{aligned}
\left(1-\beta^{2}\right) g(y)= & \frac{4 \beta^{2}}{\pi^{2}}\left[\int_{0}^{1}\left\{\frac{1}{2} y \log \left(\frac{c+t}{c-t}\right)-\frac{1}{2} t \log \left(\frac{c+y}{c-y}\right)\right\} \frac{g(t) d t}{\left(y^{2}-t^{2}\right)}+\right. \\
& \left.+\int_{0}^{1}\left\{\frac{1}{2} t \log \left(\frac{c+t}{c-t}\right)\right\} \frac{g(t) d t}{\left(c^{2}-t^{2}\right)}\right]+ \\
& +\frac{4 \beta K}{\pi^{3}}\left(T_{2}^{\infty}-T_{1}^{\infty}\right)\left(\delta_{1}-\delta_{2}\right) c y \times \\
& \times \int_{0}^{1}\{(1-s) \log (1-s)+(1+s) \log (1+s)-2 \log 2\} \\
& \times \frac{d s}{\left(y^{2}-c^{2} s^{2}\right)}+\frac{4 C_{3} A y}{\pi}, \quad 0 \leqslant y \leqslant 1 .
\end{aligned}
$$

When $g(y)$ has been found from equation (68), the complete stress and displacement field in the two half-spaces is defined through equations $(1,40$, $44,45,60,65)$. We only record here the expressions for normal and tangential traction at the crack plane. Writing $\rho=r / a$ we have

$$
\sigma_{r z}=-\frac{1}{A} \frac{d}{d \rho} \int_{\rho}^{1} \frac{g(t) d t}{\left(t^{2}-\rho^{2}\right)^{\frac{1}{2}}}, \quad 0 \leqslant \rho \leqslant 1, \quad z=0,
$$

from $(6,48)$, and

$$
\sigma_{z z}=\frac{1}{A \beta \rho} \frac{d}{d \rho} \int_{0}^{\rho} \frac{\operatorname{tg}(t) d t}{\left(\rho^{2}-t^{2}\right)^{\frac{1}{2}}}-\frac{2 C_{3}}{\beta}, \quad 0 \leqslant \rho \leqslant 1, \quad z=0,
$$

from $(61,54)$. In the range $1<\rho \leqslant c$, we substitute for $g_{1}$ from (60) to obtain

$$
\begin{aligned}
\sigma_{z z}= & \frac{2 \beta}{\pi A}\left[-\left(c^{2}-\rho^{2}\right) \int_{0}^{1} \frac{t g(t) d t}{\left(c^{2}-t^{2}\right)\left(\rho^{2}-t^{2}\right)}\right. \\
& \left.-\int_{0}^{1} \frac{\operatorname{tg}(t) \arctan \left\{\left(c^{2}-\rho^{2}\right)^{\frac{1}{2}} /\left(\rho^{2}-t^{2}\right)^{\frac{1}{4}}\right\} d t}{\left(\rho^{2}-t^{2}\right)^{\frac{1}{2}}}\right]-\frac{4 K}{\pi^{2} A}\left(T_{2}^{\infty}-T_{1}^{\infty}\right)\left(\delta_{1}-\delta_{2}\right) \times \\
& \times\left\{\frac{\pi^{2}}{8}-\chi_{2}\left(\frac{c-\left(c^{2}-\rho^{2}\right)^{\frac{1}{2}}}{c+\left(c^{2}-\rho^{2}\right)^{\frac{1}{2}}}\right)\right\} ; \quad 1 \leqslant \rho \leqslant c, \quad z=0
\end{aligned}
$$

where

$$
\begin{aligned}
\chi_{2}(x) & =\int_{0}^{x} \frac{1}{2} x \log \left(\frac{1+y}{1-y}\right) \frac{d y}{y} \\
& =\sum_{i=1}^{\infty} \frac{x^{2 i-1}}{(2 i-1)^{2}}, \quad 0 \leqslant x<1
\end{aligned}
$$

(see (8)). 
We also require the total load transmitted across the interface which is

$$
\begin{aligned}
P & =2 \pi \int_{0}^{b} \sigma_{x z}(r) d r \\
& =2 \pi \int_{0}^{b} g_{1}(t) d t,
\end{aligned}
$$

from $(60)$ on reversing the order of integration.

Substituting for $g_{1}$ from (60) and using (65), we find

$$
\begin{aligned}
P= & \frac{4 \beta a^{2}}{A} \int_{0}^{1}\left[\frac{1}{2} \log \left(\frac{c+t}{c-t}\right)+\frac{t}{\left(c^{2}-t^{2}\right)}\right] g(t) d t \\
& -\frac{4 K}{\pi A}\left(T_{2}^{\infty}-T_{1}^{\infty}\right)\left(\delta_{1}-\delta_{2}\right) a^{2} c^{2} .
\end{aligned}
$$

\section{Results for a uniform rise in temperature}

We now consider in more detail the special case in which $T_{1}^{\infty}=T_{2}^{\infty}=T_{0}$, i.e. the two bodies are both heated to the same temperature $T_{0}$ and there is no heat flow. The constant $C_{3}$ then takes the form

$$
C_{3}=\frac{\left(\alpha_{1}-\alpha_{2}\right) T_{0}}{A}
$$

and the third term in the integral equation (68) becomes zero.

The solution procedure is to regard the extent of the contact zone $(c)$ as an independent variable, solve (68) for $g(y)$ and then compute the corresponding load $P$ from (76). The kernel of the integral equation is similar to that found by Keer et al. (9) in their treatment of the penny-shaped crack and the same numerical scheme was used here. Figure 1 shows results for the load $P$ as a function of $c$ for $\beta=0 \cdot 5$. The results are plotted in terms of the non-dimensional quantity

$$
P^{*}=\frac{P A}{\left(\alpha_{1}-\alpha_{2}\right) T_{0} a^{2} \beta},
$$

which may be regarded as a ratio between mechanical and thermal effects. We also note that equation (68) for $g(t)$ depends only on $\beta^{2}$ when $T_{1}^{\infty}=T_{2}^{\infty}$ and hence the results of Fig. 1 also hold for $\beta=-0.5$. The inverse ratio $1 / P^{*}$ passes through zero for $c$ between the values 1.009695 and 1.009696 , corresponding to the purely mechanical problem of the external crack loaded in tension at zero temperature.

If temperature is now changed at constant load, the contact radius increases, decreases or remains constant if $\left(\alpha_{1}-\alpha_{2}\right) T_{0} \beta$ is positive, negative or zero respectively. Only the increasing portion of the curve can be shown in Fig. 1 because the values of $c$ corresponding to $P^{*}<0$ are very close to 1 . 


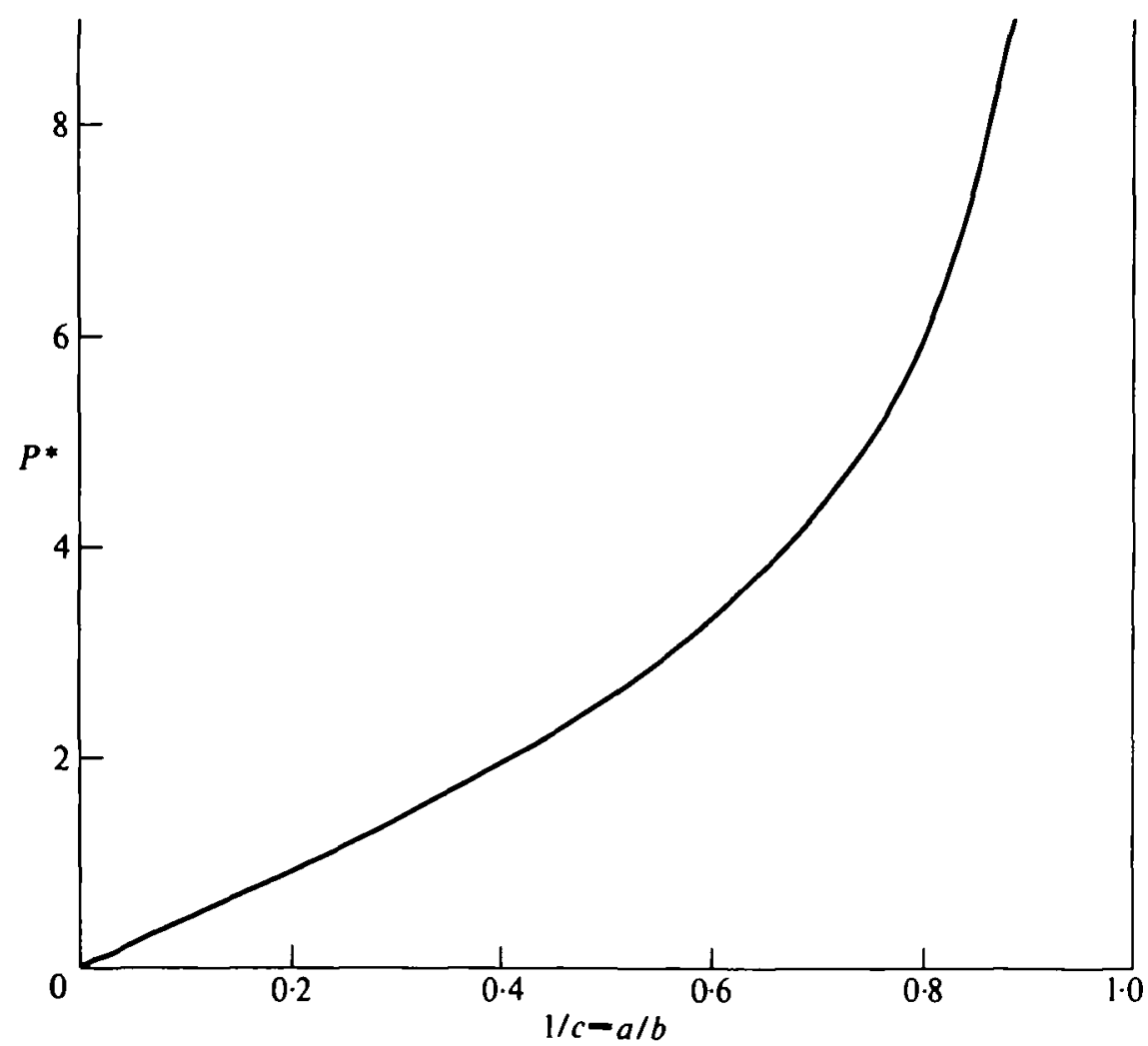

Fig. 1. Dependence of dimensionless applied load $P^{*}$ on inverse ratio of contact radii $(1 / c=a / b)$ for a uniform temperature rise $T_{0}$.

When $P^{*}$ is small (i.e. thermal effects dominate mechanical effects) the contact radius becomes inverse with $P^{*}$ and there is a limiting solution in which contact occurs over the entire unbounded region $(c \rightarrow \infty)$ and $P^{*}=0$. This corresponds to the case where there is no applied load $(P=0)$ and $\left(\alpha_{1}-\alpha_{2}\right) T_{0} \beta>0$. The solution can then be extracted analytically. For $c \rightarrow \infty$ the kernel of the integral equation (68) degenerates to zero and

$$
\left(1-\beta^{2}\right) g(y)=\frac{4 C_{3} A y}{\pi}, \quad 0 \leqslant y \leqslant 1 .
$$

Substituting into equations $(69,70,71)$ gives

$$
\begin{gathered}
\sigma_{r z}=\frac{4\left(\alpha_{1}-\alpha_{2}\right) T_{0}}{\pi A\left(1-\beta^{2}\right)} \frac{r}{\left(a^{2}-r^{2}\right)^{1}}, \quad 0 \leqslant r \leqslant a, \\
\sigma_{z z}=\frac{2\left(\alpha_{1}-\alpha_{2}\right) T_{0} \beta}{A\left(1-\beta^{2}\right)}, \quad 0 \leqslant r \leqslant a, \\
\sigma_{z x}=\frac{4\left(\alpha_{1}-\alpha_{2}\right) T_{0} \beta}{\pi A\left(1-\beta^{2}\right)}\left[\frac{a}{\left(r^{2}-a^{2}\right)^{\frac{1}{2}}}-\arcsin \frac{a}{r}\right], \quad r>a .
\end{gathered}
$$




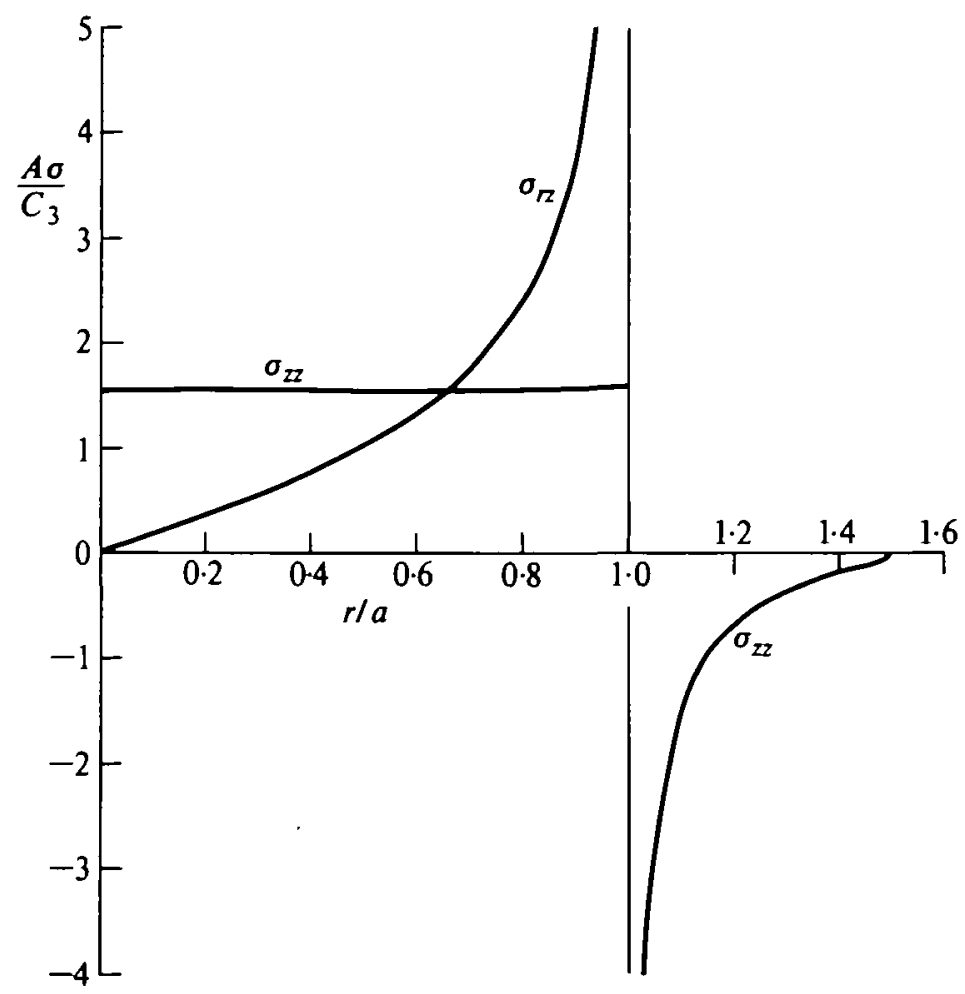

Fig. 2. Tractions at the interface for $c=1 \cdot 5$.

Thus, the normal traction is a constant tension in the bonded region and a compressive traction singular as $r \rightarrow a+$ in $r>a$. The shear traction is also singular as $r \rightarrow a-$.

We would anticipate a similar limiting solution for $\left(\alpha_{1}-\alpha_{2}\right) T_{0} \beta<0$ with a contact radius ratio very close to unity. However, the computational techniques employed make it impossible to extract this limit. $P^{*}$ increases monotonically through negative values in $c<1.009696$, but at $c=1.0000001$ we still have $P^{*}=-9 \cdot 5$.

The distribution of tractions on $z=0$ has been computed for other values of $c$ and the results for $c=1.5$ are given by way of illustration in Fig. 2.

\section{Examples with heat flow}

If the temperatures $T_{1}^{\infty}, T_{2}^{\infty}$ are unequal, causing heat to flow between the bodies, there is no dimensionless presentation which preserves generality. We therefore restrict attention to the special case in which body 2 is a rigid perfect conductor $\left(\mu_{2}, K_{2} \rightarrow \infty, \alpha_{2}=0\right)$ at temperature $T_{0}$, whilst $T_{1}^{\infty}$ is zero. 
The free term in equation (68) then takes the form $\frac{2 \alpha_{1}}{\pi}\left(1+\nu_{1}\right) T_{0} \times$ $\times\left[y+\frac{2 \beta c}{\pi} \int_{0}^{1}\{(1-s) \log (1-s)+(1+s) \log (1+s)-2 \log 2\} \frac{d s}{\left(y^{2}-c^{2} s^{2}\right)}\right]$.

Figure 3 shows the variation of applied load $P$ (tensile positive) with $1 / c$ $(=a / b)$ for $\beta=0.5$, which here corresponds to $\nu_{1}=0$.

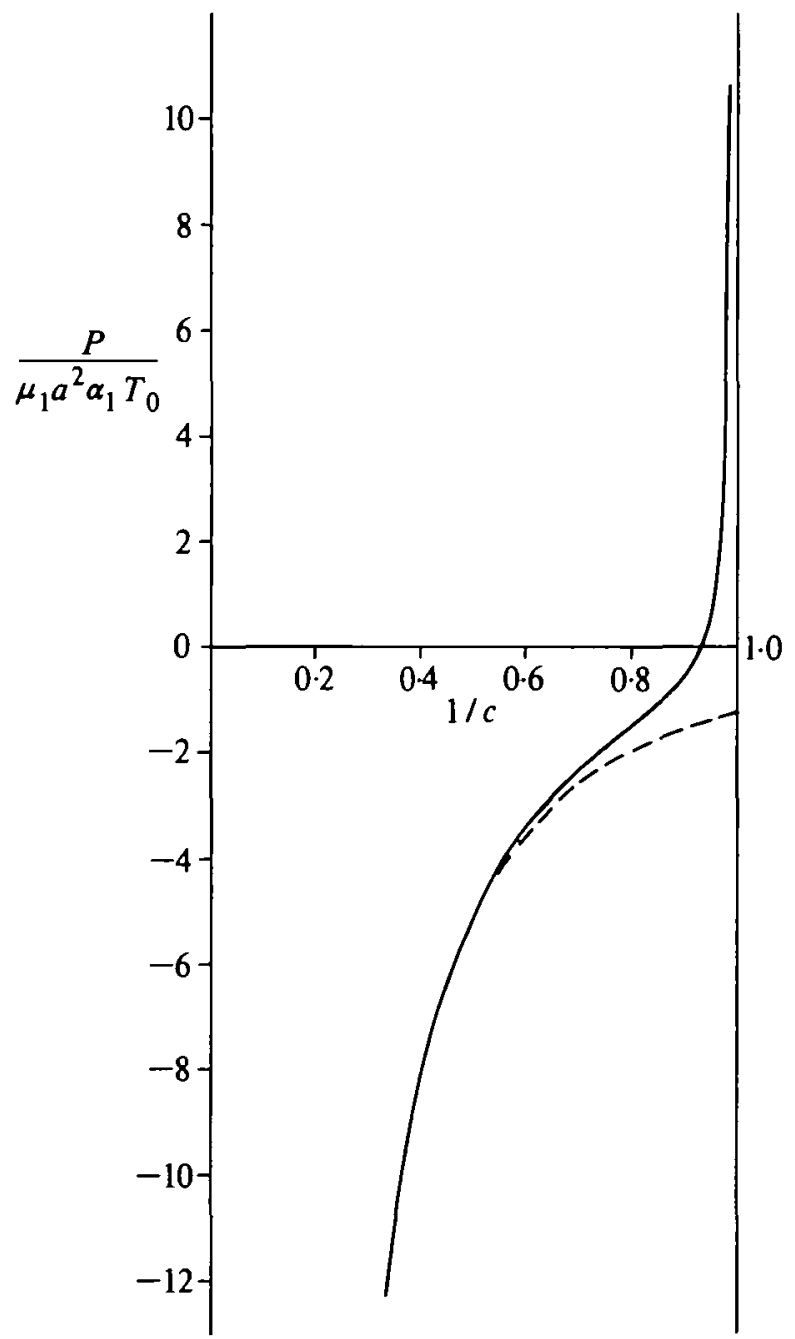

FIG. 3. Applied load $P$ as a function of contact radius ratio $(1 / c=a / b)$ with one body a rigid perfect conductor. 
As in the previous example, the solution for the external crack with purely mechanical loading is recovered when $P \rightarrow+\infty$. At the other extreme when $P$ is large and negative, a large contact region is developed. The bonded region has then a relatively small effect on $P$ and the latter approaches the solution for the corresponding frictionless contact problem (10),

$$
P=-\frac{4 \mu_{1} \alpha_{1}\left(1+\nu_{1}\right) T_{0} b^{2}}{\pi\left(1-\nu_{1}\right)}
$$

which is shown dotted in Fig. 3.

If the bodies are subjected to a heat flux but no load $(P=0)$ we find $c=1.0743$.

A more extensive study of the effect of thermal and mechanical conditions and material properties will be published elsewhere.

\section{Ackmowledgement}

The authors are pleased to acknowledge support for this research by the U.S. Army Research Office through Grant Number DAAG29-82-K-001.

\section{REFERENCES}

1. M. Comninou, J. appl. Mech. 44 (1977) 631-636.

2. - Int. J. engng Sci. 18 (1980) 501-506.

3. J. R. BARBer, J. Elast. 8 (1978) 409-423.

4. A. E. Green and W. Zerna, Theoretical Elasticity (Clarendon Press, Oxford, 1954) 174-178.

5. J. R. BARBER, The Mechanics of the Contact between Deformable Bodies (Eds. A. D. de Pater and J. J. Kalker) (Delft University Press, 1975) 177-190.

6. W. D. Collins, Proc. Edin. math. Soc. 13 (1963) 235-246.

7. J. Dundurs, J. appl. Mech. 36 (1969) 650-652.

8. L. LewiN, Dilogarithms and Associated Functions (MacDonald, London, 1958).

9. L. M. Keer, S. H. Chen and M. Comninou, Int. J. engng Sci. 16 (1978) $765-772$.

10. J. R. BARBer, Int. J. mech. Sci. 15 (1973) 813-819. 